Jingxin Su*, Zhiping Wang, Tianjing Wang, Aizhen Ji, Liguo Hou, Xiaomei Guo

\title{
Influence of Atmosphere Environment on Aircraft Heat Exchanger Fouling Growth Characteristics
}

\begin{abstract}
During domestic civil aviation operation, problems of fouling on the cooling surface of the aircraft heat exchanger have been more serious than ever due to air pollution. By using analytical methods including energy dispersive spectroscopy (EDS), energy dispersive X-ray (EDX) and ion chromatography respectively, sources of aircraft air-conditioning heat exchanger dirt are verified to be mainly air pollutants and soil particles. And by observing dirt growth characteristics under different surface states, it's found that when there are large particles such as catkins on the surface of samples, the rate of fouling is increased for the reason that large particles will augment adsorption sites of nucleation. It turns out that dirt nuclei selectively concentrate in the vicinity of large particles instead of generating randomly. While oil and residual oily cleaning agents can inhibit the growth of fouling by isolating test pieces from atmosphere.
\end{abstract}

Keywords: aircraft heat exchanger, thin film, crystallization fouling, nucleation sites.

\section{Introduction}

Heat exchanger is an important component of the aircraft air-conditioning system, whose reliability plays a decisive role for the reliability of the whole aircraft environmental control system. There are different degrees of fouling problems for most of the heat exchangers, wherein its fouling problem on cooling surface has been a widespread problem during domestic civil aircraft operation. The air conditioning system failure rate of Boeing 737, the main trunk of most airlines, is relatively high, especially in hot summer. Faults often appear one after another, which not only influence line maintenance, aircraft release and other produces, but also affect the operating efficiency and maintenance costs of the whole aviation business [1]. Once the working efficiency of heat exchanger effect is so poor that cold airflow temperature reaches any protection temperature, thermal protection

\footnotetext{
*Corresponding author: Jingxin Su, Tianjin Key Laboratory for Civil Aircraft Airworthiness and Maintenance, Civil Aviation University of China, Tianjin, China, E-mail: sujingxin@139.com

Zhiping Wang, Aizhen Ji, Tianjin Key Laboratory for Civil Aircraft Airworthiness and Maintenance, Civil Aviation University of China, Tianjin, China

Tianjing Wang, Liguo Hou, Xiaomei Guo, Aircraft Maintenance and Engineering Corporation, Beijing Capital International Airport, Beijing, China
} 
system will automatically shut off assembly valve and the air conditioning system will stop working [2].

Current research focuses more on fault repair, rather than develop into the root causes of failure. Therefore, understanding the mechanism of the formation and growth characteristics of dirt, determining cleaning cycle and optimizing cleaning process according to the characteristics of different growth stages of dirt become more important. From hydro-dynamics and physicochemical conditions two viewpoints, Yiantsions [3] analyzed the deposition process of micron particles in the heat exchanger surface, whose experimental results reveal the interaction between gravity and other physical and chemical factors. By assuming crystallization dirt and particles dirt exist independently, crystallization occurs simultaneously on the heat exchanger surface and the particle surface as well. Xu Zhiming [4] used the area ratio to divide calcium carbonate deposition into two parts: Part of the calcium carbonates are deposited on the wall, forming a crystallization fouling; another part of the calcium carbonate particles deposit on the surface of particles dirt, joining deposition process of particles dirt. Zhang Zhongbin [5] studied the effects of heat transfer surface characteristics and working fluid flow characteristics on fouling induction period by the method of comparative experiments, results showed that fouling induction period of the heat transfer was extended with the reduction of material surface adhesion and the enhance of field disturbance. Roya Sheikholeslami [6] demonstrates that other non-crystallizing species, if present, may affect the surface energy of a crystal and alter the Gibbs free energy for homogeneous nucleation and other crystallizing species may also act as a nucleus initiating nonhomogeneous nucleation with a lower Gibbs free energy and promoting nucleation.

In order to observe the growth characteristics of crystallization fouling, Won Tae Kim [7] used microscopy to observe the growth of $\mathrm{CaCO}_{3}$ crystallization fouling, whose experimental device was designed to observe the growth process of dirt in a sealed system, which cannot be used very well to study the growth of crystallization dirt under open system.

In this paper, analytical methods including EDS, EDX and ion chromatography are used to verify the source and main ingredients of aircraft air-conditioning heat exchanger. Using the analytical results obtained, with reference to thin film research methods, we study the crystallization fouling growth characteristics of aerosol whose main soluble ions are $\mathrm{Cl}^{-}, \mathrm{NH}_{4}^{+}, \mathrm{NO}_{3}{ }^{-}, \mathrm{SO}_{4}^{2-}$ and $\mathrm{Na}^{+}$under humid atmosphere. Soluble salt mixed solution was prepared to form a thin film on the specimen surface and placed in the simulated work environment. The growth of the dirt was under open systems, using microscopic observation for the growth process of air side fouling. 


\section{Experimental Methods}

\subsection{Aircraft Air-conditioning Heat Exchangers Pollution Analysis}

According to their present status, air pollutants can be classified as aerosols pollutants and gaseous status pollutants. Aerosols pollutants mainly include dust, mist, fly ash and suspended solids; Gaseous pollutants include sulfur oxides, nitrogen oxides, carbon oxides and hydrocarbons.

The atmospheric aerosol, referring to the multi-phase systems composed of the atmosphere and the solids and liquid particles suspended in it, which includes Total Suspended Particles (TSP), particles with an aerodynamic diameter less than or equal to $100 \mu \mathrm{m}$ suspended in the atmosphere and water-soluble ions. Water-soluble ionic components $\mathrm{SO}_{4}^{2-}, \mathrm{NO}_{3}, \mathrm{Cl}^{-}, \mathrm{NH}_{4}^{+}, \mathrm{K}^{+}, \mathrm{Na}^{+}, \mathrm{Ca}^{2+}$ and $\mathrm{Mg}^{2+}$ account for a large proportion in atmospheric aerosols. In recent years, Chinese environmental scientists have made a lot of progress in the study of atmospheric particulates [8]. It summarizes the most common source of part ions in aerosols as shown in Table 1.

Table 1: Common Source of Part lons in Aerosols.

\begin{tabular}{ll}
\hline Ions & Common Source \\
\hline $\mathrm{SO}_{4}{ }^{2-}$ & Combustion of coal, oil and other fossil fuels \\
$\mathrm{NO}_{3}{ }^{-}$ & Oxidation of $\mathrm{NO}_{\mathrm{x}}$ produced by combustion of fossil fuel and natural gas \\
$\mathrm{Cl}^{-}$ & Combustion of waste garbage, industrial production \\
$\mathrm{NH}_{4}^{+}$ & Farming and animal husbandry, such as rotting plants, animal wastes and fertilizer use \\
$\mathrm{K}^{+}$ & Emission of soil or human life combustion \\
$\mathrm{Na}^{+}$ & Form of NaCl \\
$\mathrm{Ca}^{2+}$ & Soil dust and construction \\
$\mathrm{Mg}^{2+}$ & Soil dust \\
\hline
\end{tabular}

Due to the large surface area, it is easy for small particles to absorb plenty of organics on its surface [9]. Therefore, the organic pollutants are also an important component of air pollution. Particulate organic matters are composed of hundreds of organic compounds, with widely varied chemical properties and thermodynamic properties, which bring a lot of difficulties for the study [10]. But through the accumulation of research, environmental scientists achieved certain results on the chemical composition and characteristics of particulate organic matter. Organic pollutants in the atmosphere mainly come from direct emissions, including incomplete combustion of fossil fuels and biomass, source plant emissions and natural fire, while some of it is the secondary source generated by chemical reaction of gaseous organic compounds [11]. 


\subsection{Dirt Collection of Airborne Heat Exchangers}

After theoretical analysis of the heat exchanger fouling sources, we understand the possible composition of dirt. And to further verification, the analysis of the dirt collection through the heat exchanger is needed. Direct collection from the heat exchanger surface not only can destroy the heat exchanger, but also the amount of dirt obtained is so small that it is not easy to analyze its composition. Therefore, in this paper, we collected dirt from heat exchanger by indirect methods and analyzed it.

The performance of contaminated heat exchanger will degrade which can easily lead to failure of the air conditioning system, thus the heat exchanger is periodically cleaned. During the cleaning process, the cleaning agent will dissolve part of the dirt which reduces the binding forces between dirt, resulting in some dirt off. This paper analyzes the insoluble dirt off particles during the cleaning process of heat exchanger. Separating insoluble dirt particles from the cleaning agent by using high-speed centrifuge, after which dirt particles were put in a drybox under $80^{\circ} \mathrm{C}$ for $24 \mathrm{~h}$ to obtain powdery particles of dirt. Analysis of the powder can be done by means of fluorescence spectroscopy to obtain elemental composition and mass fraction of insoluble dirt.

In addition, cut the collected waste heat exchanger to observe the cold road fin within it. Using scanning electron microscopy to observe the dirt on the surface of fin and X-ray spectroscopy to analyze the elemental composition, elemental composition of dirt on fin surface can then be obtained.

The results obtained by the two experimental programs above are just the elemental composition of dirt, but not the direct expression of dirt components. The working principles of aircraft heat exchanger and outdoor heat exchanger are the same, of which the main sources of dirt are aerosols pollutants and particulates. So in order to be more intuitive for the understanding of the composition of dirt, this experiment has collected dirt on the heat exchanger after perennial work outdoors. Immersed in deionized purified water for $240 \mathrm{~h}$, separate the insoluble dirt particles by centrifugation and analyze the solution by ion chromatographic method to detect the soluble ions in it. Dirt sources, analytical procedures and the applicability of methods are all in Table 2.

Table 2: Part of Soluble Ions in Aerosols.

\begin{tabular}{lll}
\hline Sources of Dirt Collection & Analytical Procedures & Applicability of Methods \\
\hline $\begin{array}{l}\text { Insoluble particles obtained by } \\
\text { centrifugation of cleaning agent }\end{array}$ & EDX & $\begin{array}{l}\text { Analysis of elemental composition } \\
\text { and percentage of weight of the } \\
\text { particles } \\
\text { Dirt on the surface of heat exchanger }\end{array}$ \\
& EDS & $\begin{array}{l}\text { Analysis of elemental composition } \\
\text { and percentage of weight of the } \\
\text { particles }\end{array}$ \\
$\begin{array}{l}\text { Outdoor air conditioning heat } \\
\text { exchanger }\end{array}$ & Ion Chromatography & $\begin{array}{l}\text { Analysis of soluble anion and cation } \\
\text { in solution }\end{array}$ \\
\hline
\end{tabular}




\subsection{Crystallization Growth Characteristics under Thin Film Environment}

The experiments use a self-designed thin film equipment to study crystallization growth characteristics, as shown in Fig. 1. Wherein Fig. 1a is a principle diagram of thin film device and Fig. 1b is a front view of the thin film device. Thin film device consists of thin liquid film work systems, thickness measuring system and observation system. Thin film measuring device [12] is mainly composed by ohmmeter and screw micrometer equipped with fine platinum needle.

When measuring the thickness of thin film, fix the screw micrometer stuck with platinum needle directly above the test piece and adjust the micrometer screw slowly and carefully to enable platinum needle close to the specimen surface. When platinum needle tip gets in contact with the electrode surface, the ohm resistance shown in the table jumps from the infinite to a fixed value, then stop the adjustment of screw micrometer immediately and read the screw micrometer scale, denoted by $h_{1}$. Then getting the platinum needle back away from the test piece surface, the deionized water flowed through the inlet sweep over the whole specimen. Now adjust the screw micrometer again to get the platinum needle close to the surface of the solution until they are in contact, read screw micrometer scale $h_{2}$, so that the thickness of the film may be referred to as $\triangle h=h_{2}-h_{1}$. The operating temperature of aircraft air-conditioning heat exchanger cold road is usually stable at $80^{\circ} \mathrm{C}-120^{\circ} \mathrm{C}$, over which it will start selfprotection and shut down the system, so this experiment controls the temperature of thin film device at $80^{\circ} \mathrm{C}$ by heating station. Close pure water flows after that deionized pure water sweep over the specimen, with the thin film device at $80^{\circ} \mathrm{C}$, pure water will evaporate which decreases thin film thickness. At this time since solution is deionized pure water, there will be no precipitation of dirt at this stage called preparation of thin film. Continuously measuring the value $h_{2}$, open the peristaltic pump when $\triangle h$ is less than $300 \mu \mathrm{m}$, letting the dirt solution flow into the thin film in accordance with a certain velocity. By controlling the flow rate of the peristaltic pump, the thin film can be stabilized at $200 \mu \mathrm{m}-300 \mu \mathrm{m}$. After repeated experiments, we found that when the inlet flow rate is controlled between $320 \mu \mathrm{L}$ and $350 \mu \mathrm{L}$, the thin liquid film thickness can be controlled at $200 \mu \mathrm{m}-300 \mu \mathrm{m}$. Thereafter, the thickness measuring system can be replaced by observation system, photographing at a certain time to observe the growth of fouling.

In order to ensure that the dirt precipitation can be accelerated, in this study, mixed solution of $\mathrm{NH}_{4} \mathrm{Cl}, \mathrm{Na}_{2} \mathrm{SO}_{4}$ and $\mathrm{NaNO}_{3}$ with $80 \%$ molar concentration respectively are prepared as the analog solution. Under actual operating conditions, the surface of the heat exchanger does not always maintain the clean state, but there will be a wide variety of dirt adsorbing on the surface, so the surface of the test sample was divided roughly into four categories for analysis: without any treatment, that is, clean state; catkins attached to the surface which characterize the state of large particles adsorption on the heat exchanger surface; attached to aviation kerosene, showing the state of a heat exchanger attached with organic pollutants in the atmosphere; 
with cleaning agent, that is when the heat exchanger wasn't washed clean. Therefore the experiments were divided into four groups wherein the sample surface of the first group received no treatment. And during thin film preparation process of second group, catkins will be spread evenly in thin liquid film surface. In front of the thin film preparation process of the third and fourth group, aviation kerosene and Turco3878LF-NC cleaning agent coated evenly on specimen surface. After that, start thin film preparation until it achieves the demanded degree, then open peristaltic pump, dirt solution slowly flowing on thin liquid film surface by a certain velocity.

(a) Thin film device operating principle

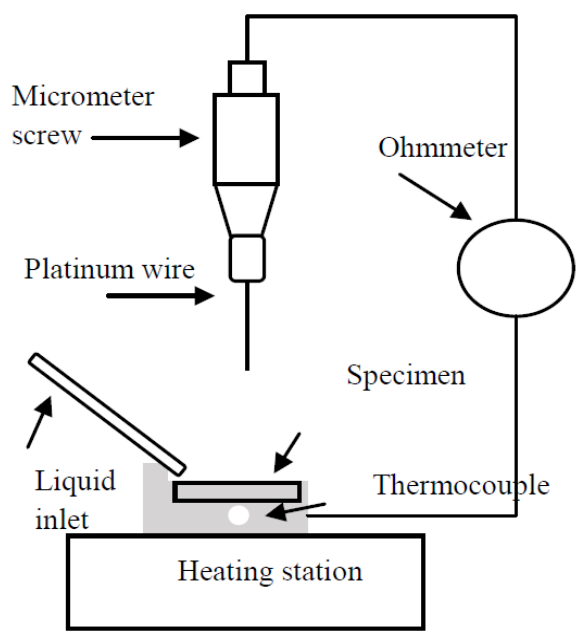

(b)Thin film device front view

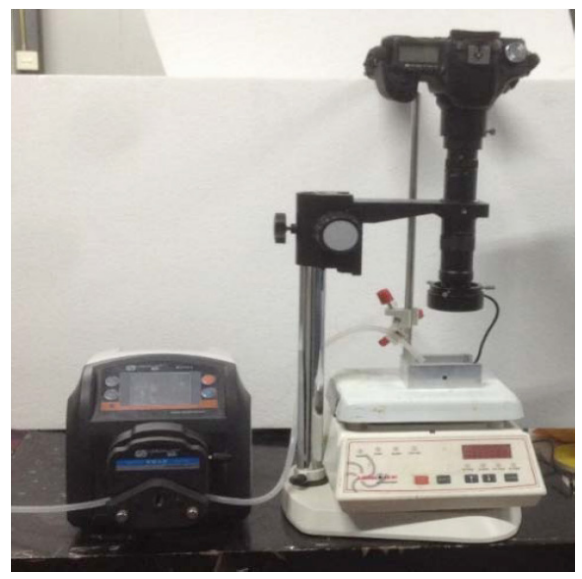

Figure 1: Designed thin film equipment

\section{Results}

\subsection{Main Ingredients of Aircraft Air-conditioning Heat Exchangers}

By taking a small portion of the fin from the abandoned aircraft heat exchanger surfaces, we use scanning electron microscopy X-ray energy dispersive spectroscopy to analyze surface dirt micro-element composition. Analysis results are shown in Fig. 2 and Table 3. Collecting waste liquid of heat exchanger after cleaning from an aircraft maintenance company, insoluble dirt is obtained by centrifugation. Elemental composition analysis can be done by EDX, the results are shown in Table 3.

As it can be seen from Table 3, major elements in dirt are $\mathrm{Na}, \mathrm{Mg}, \mathrm{Si}, \mathrm{K}, \mathrm{Ca}, \mathrm{Fe}, \mathrm{Cu}$ and $\mathrm{O}$, which are common elements in the atmosphere or soil particles. The insoluble 
particles obtained by centrifugation can be observed by a laser microscope, shown in Figure 3. Insoluble particles in dirt are mainly various colors of minerals, of which main source are soil particles, further validating the foregoing analysis about dirt sources of heat exchanger. And through the ion chromatographic analysis about outdoor heat exchanger, main soluble ions: $\mathrm{Na}^{+}, \mathrm{NH}_{4}^{+}, \mathrm{K}^{+}, \mathrm{Mg}^{2+}, \mathrm{Ca}^{2+}, \mathrm{Cl}^{-}, \mathrm{NO}_{2}, \mathrm{Br}$, $\mathrm{NO}_{3}{ }^{-}$and $\mathrm{SO}_{4}{ }^{2-}$, which is very close to the elemental analysis results of aircraft airconditioning heat exchanger. In conclusion, the source of aircraft air-conditioning heat exchanger dirt are mainly air pollutants and soil particles.

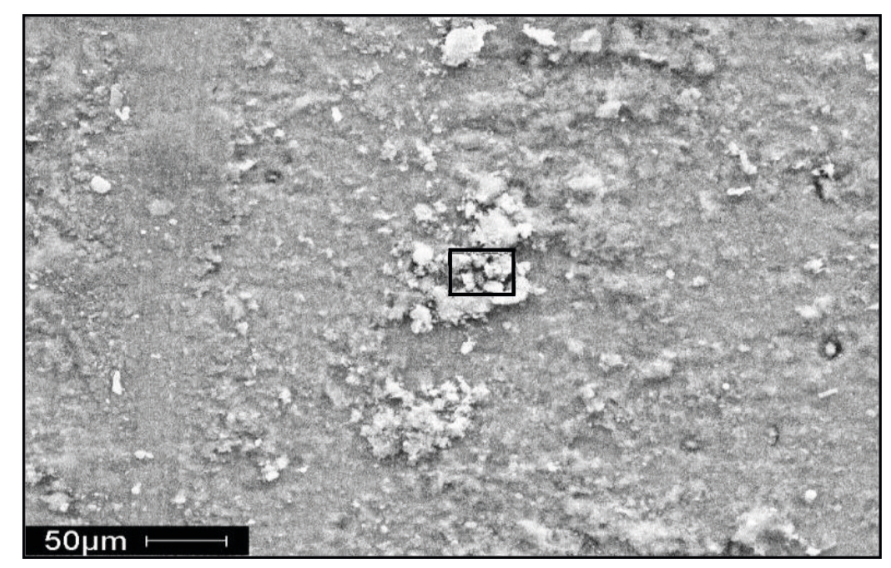

Figure 2: SEM laser spectroscopy bit
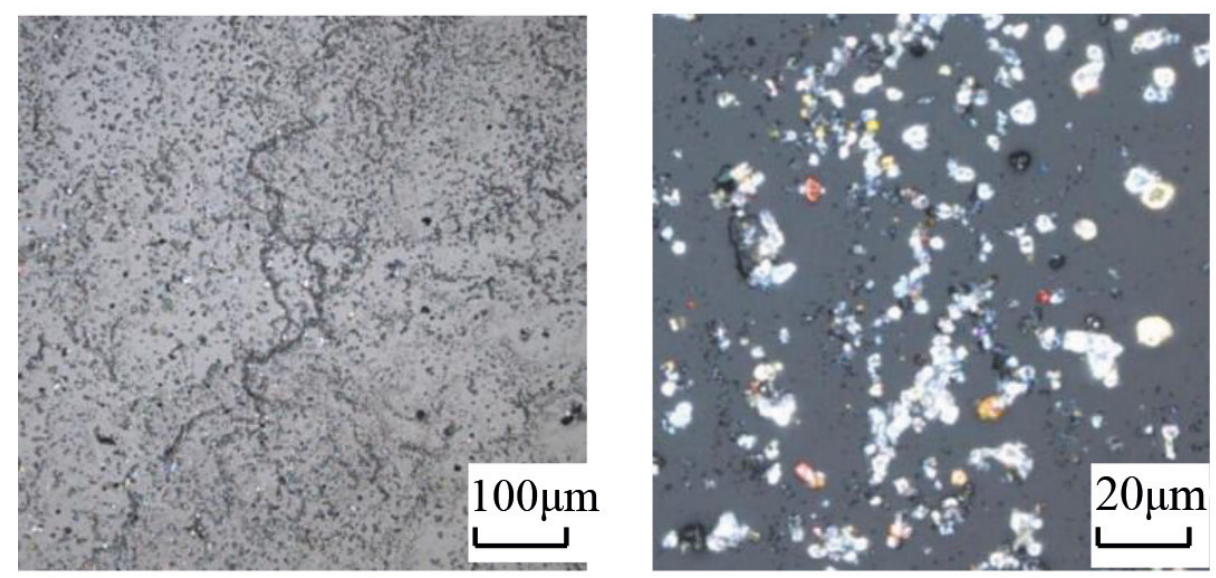

Figure 3: Insoluble dirt particle morphology under a laser microscope 
Table 3: Analysis of Results of Dirt Sample Elements

\begin{tabular}{lllllllll}
\hline Element & $\mathbf{0}$ & $\mathrm{Na}$ & $\mathbf{M g}$ & $\mathbf{A l}$ & $\mathbf{S i}$ & $\mathbf{P}$ & $\mathbf{S}$ & $\mathbf{K}$ \\
\hline EDS & 19.93 & 2.24 & 2.98 & 42.61 & 3.87 & 2.31 & 1.22 & 1.28 \\
EDX & - & 7.283 & 2.288 & 11.96 & 27.17 & 3.87 & - & 4.385 \\
Element & $\mathrm{C}$ & $\mathrm{Ti}$ & $\mathrm{Cl}$ & $\mathrm{Ni}$ & $\mathrm{Co}$ & $\mathrm{Ca}$ & $\mathrm{Fe}$ & $\mathrm{Cu}$ \\
EDS & - & - & - & - & - & 1.37 & 20.19 & 1.82 \\
EDX & 24.8 & 1.36 & 0.665 & 0.403 & 0.0226 & 2.863 & 12.2 & 0.122 \\
\hline
\end{tabular}

\subsection{Weight Gain Curve and Surface Topography of Crystallization}

Fig. 4 is the crystallization fouling weight gain curve under different surface states, which shows that the fastest growing of crystallization fouling is when large dirt particles such as catkins adhesion on the surfaces whereas dirt growth has been somewhat suppressed when there are oil or residual cleaning agents on the surface. This is because the presence of oil and oil-based cleaning agents specimen surface prevent the thin film from direct contact with the test piece so that after the formation of dirt nucleation, it cannot be adsorbed on the test piece surface but floating on oil surface, only large particles of dirt can be deposited and adsorbed onto the surface of the test piece. Dirt nucleation floating on the oil surface are easily taken away by flowing thin film, it is not easy to complete this series of nucleation and growth process in the test piece surface.

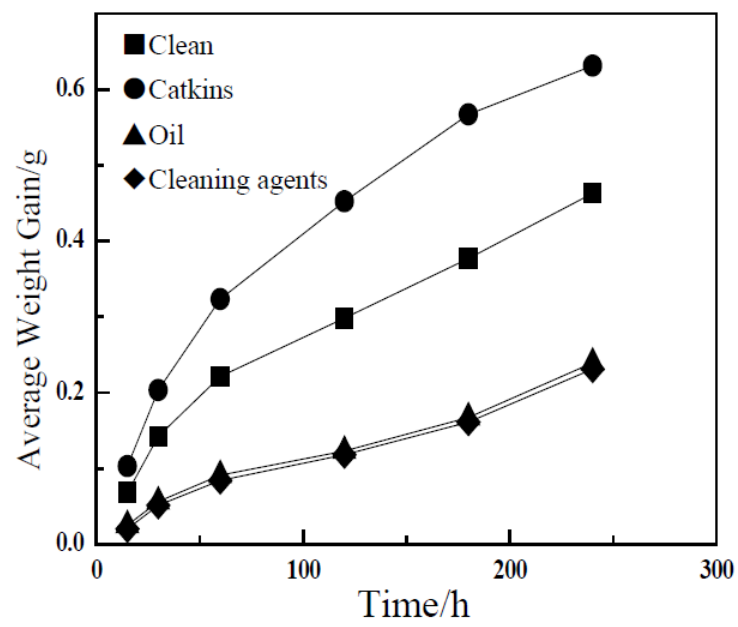

Figure 4: Crystallization fouling weight gain curve under different surface states 
To further study the differences of crystallization fouling, the sample surface topography at different times are taken during the testing process as shown in Fig. 5-8. Fig. 5 shows the growth process of dirt on the surface of flat specimen polished using the \#1200 sandpaper. Some scholars have studied the influence of different surface roughness on fouling growth performance, founding that dirt adsorption on the surface of the sample exhibits random results [13].

Therefore, this paper does not consider the effect of roughness on the growth of fouling. During the experiment, on the thin film a lot of small bubbles appeared randomly, as shown in Fig. 5b. As the bubbles escaped, local concentration increased and salt crystal nucleation was formed here. Deposited on the test piece surface, part of dirt nucleation was taken away by the flowing thin film, while others which were adsorbed on the surface of the sample continued to grow, as shown in Fig. 5c. The form of deposited dirt is like dendrite and the staggered growth gives them a strong binding. Bubble randomness also illustrates absorption in the dirt is with certain randomness.
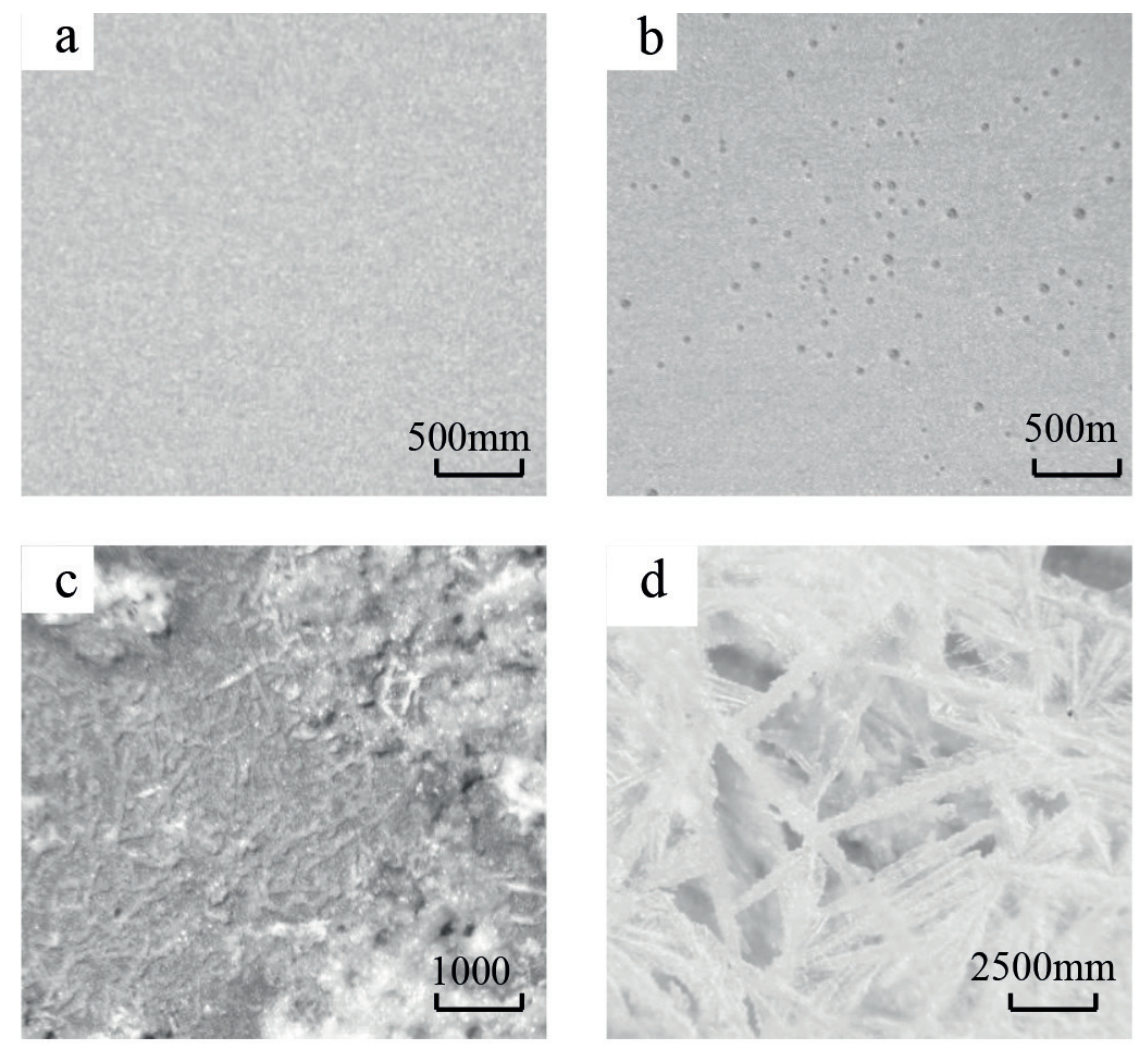

Figure 5: Topography of sample with clean surface over time, a: 0 h; b: 0.5 h; c: 2 h; d: 4 h 
Fig. 6 illustrates dirt growth characteristics when catkins attached to the surface. As can be seen from Fig. 6b, the bubbles don't occur randomly, but selectively concentrated in the vicinity of catkins. This is because when there are catkins and other solids in a thin film, the surface energy is reduced, so that air bubbles in the thin film will be attached and accumulated on the surface of catkins. Large bubbles formed by the accumulation of small bubbles can easily escape from the thin film, increasing local concentration. Dirt nucleation does not need to be on the sample surface, but grows in the surface of catkins. Besides, additional crystal nuclei can significantly improve crystallization rate effects [14-15]. Meanwhile, the nuclei adsorbed on catkins are not likely to be taken away by flowing thin liquid film, so dirt growth was relatively rapid. And after that, catkins were firmly adsorbed on the surface of the test piece, easy to form stubborn dirt, as shown in Fig. 6c. As can be seen from Figure $6 \mathrm{~d}$, the presence of catkins made the binding of dirt more compact, on the one hand increased the absorption point of dirt nucleation, and on the other side increased the binding ability between dirt.
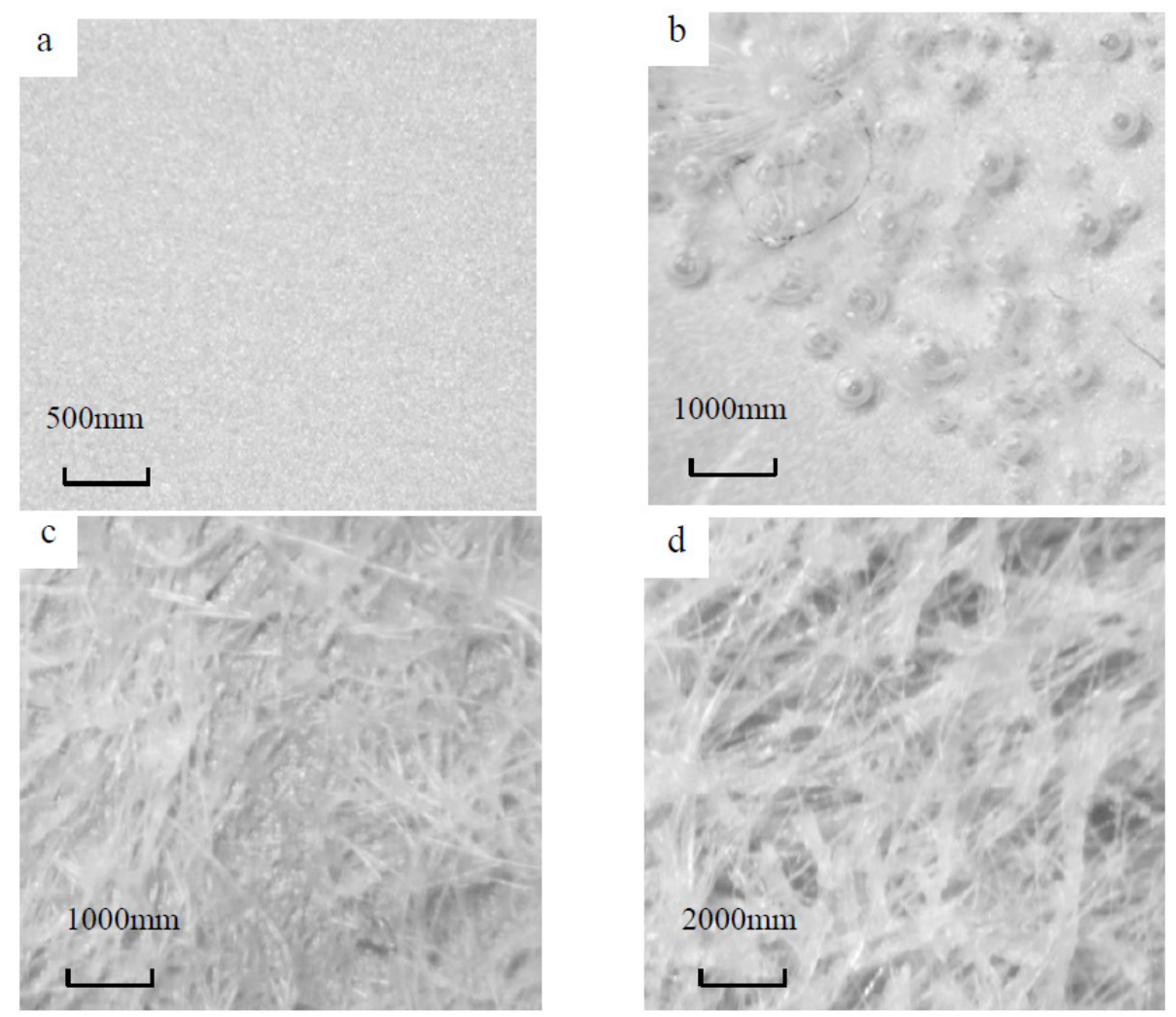

Figure 6: Topography of sample surface with catkins attachment over time, a: 0 h; b: 0.5 h; c: 1 h; d: 2 h 
Fig. 7 shows the existing form when there are oils on the surface of the sample. As can be seen from Fig. 7b, dirt is wrapped by oil, only heavier dirt could deposit beneath the oil layer to be in contact with the test piece in order to complete this phase of growth, verifying the above analysis about that dirt gains weight slower than others with oil on the surface.
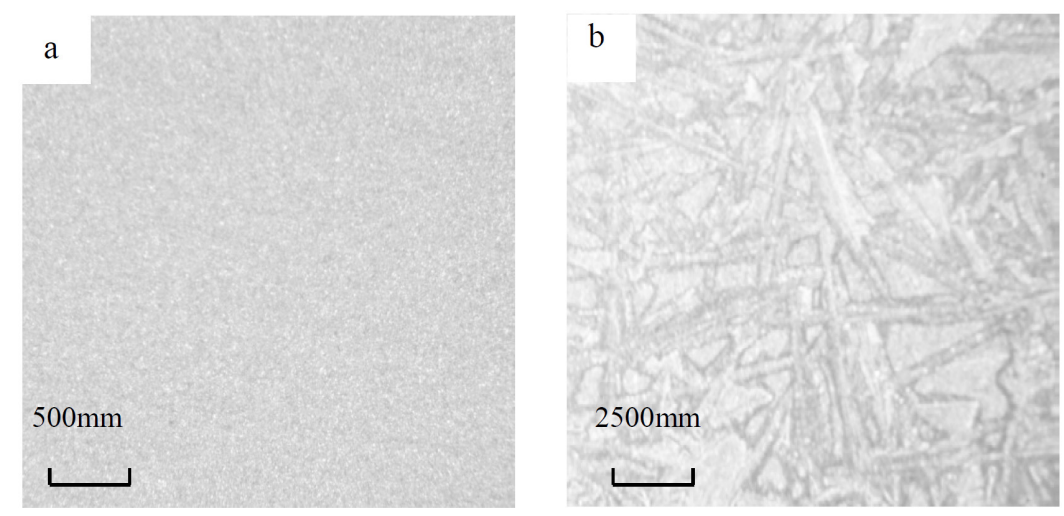

Figure 7: Topography of sample surface with oil attachment over time, a: 0 h; b: $4 \mathrm{~h}$

Fig. 8 illustrates the change with time of sample surface covered with cleaning agent. Dirt is wrapped by oily cleaning agent as shown in Fig. 8b, which demonstrates that the inhibition of dirt by cleaning agent is also because of the cut of contact between the thin film and the test pieces. At the same time, dirt precipitated from the cleaning agent is relatively small compared to other dirt, indicating that the cleaning agent has a potent inhibitory effect on dirt growth.
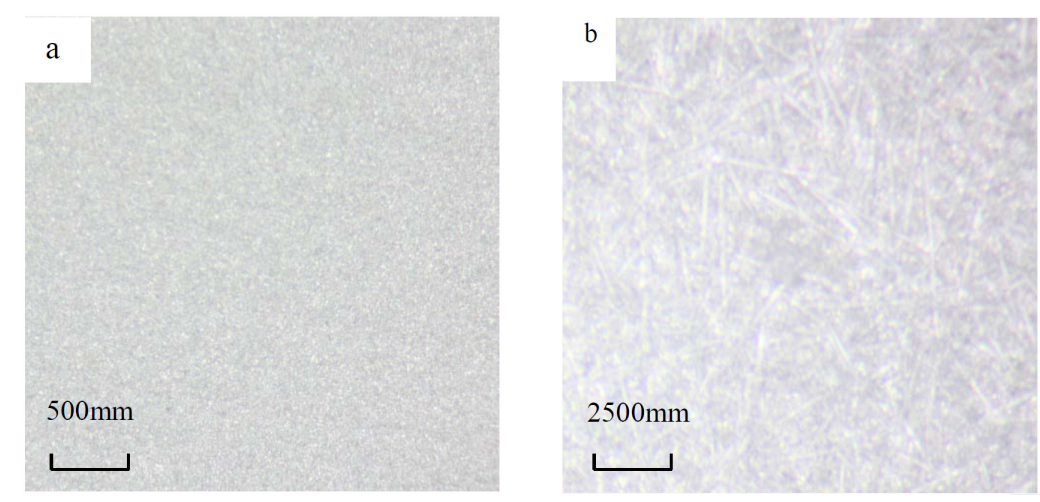

Figure 8: Topography of sample surface with cleaning agents over time, a: $0 \mathrm{~h}$; b: $4 \mathrm{~h}$ 


\section{Conclusions}

The source of aircraft air-conditioning heat exchanger dirt are mainly air pollutants and soil particles, including ions such as $\mathrm{Na}^{+}, \mathrm{NH}_{4}^{+}, \mathrm{K}^{+}, \mathrm{Mg}^{2+}, \mathrm{Ca}^{2+}, \mathrm{Cl}^{-}, \mathrm{NO}_{2}, \mathrm{Br}, \mathrm{NO}_{3}$ and $\mathrm{SO}_{4}^{2}$.

During the evaporation process, dirt nuclei generated randomly in the thin film. But when the test piece surface has catkins or other large particulates, it will augment adsorption sites of nucleation. Thereby the growth of dirt was significantly increased, the density of the dirt as well.

When there are large particles like catkins on the surface of specimens, the crystallization dirt grows the fastest, while when the specimens are covered with oil or residual cleaning agents, fouling growth has been somewhat suppressed for the reason that residual cleaning agents and oil prevent test piece from the atmosphere, it's difficult for the dirt nucleation to deposit on the surface of the sample.

Acknowledgment: This study was supported by the Major Science \& Technology Research Project of Civil Aviation of China (No. MHRD20140110).

\section{References}

[1] Chen M.Y., Zhang X.Y., Cai W., Operating Analysis of Airplane Air Conditioning Heat Exchanger[J]. J. Shanghai Univ. Eng. Sci., 2008, 04:310-314

[2] Li L.K., Shooting of “B737-300/500 A/C System Cannot Cooling”[J], Aviat. Maint. Eng., 2004, 3:48-49

[3] Yiantsios S.G., Karabelas A.J., Deposition of micron-sized particles on flat surfaces: Effects of hydrodynamic and physicochemical conditions on particle attachment efficiency[J], Chem. Eng. Sci., 2003, 58(14):3105-3113

[4] Xu Z.M., Zhang Z.B., Guo W.Z., et al., A theoretical model of composite fouling of particulate and crystallization[J], J. Eng. Thermophys., 2006, 27(z2):81-84

[5] Zhang Z.B., Xu Z.M., Qiu Z.B., Experimental investigation on effect of surface and flow characteristics on the induction period of fouling[J], J. Eng. Thermophys., 2009, 30(1):144-146

[6] Sheikholeslami R., Nucleation and kinetics of mixed salts in scaling[J], Aiche J., 2003, 49(49):194-202

[7] Kim W.T., Bai C., Cho Y.I.,A study of $\mathrm{CaCO} 3$ fouling with a microscopic imaging technique[J], Int. J. Heat Mass, 2002, 45(3):597-607

[8] Yang F.,Tan J.,Zhao Q., et al., Characteristics of $\mathrm{PM}_{2.5}$ speciation in representative megacities and across China[J], Atmos. Chem. Phys., 2011, 11: 5207-5219

[9] Ravishankara A.R., Heterogeneous and Multiphase Chemistry in the Troposphere[J], Science, 1997, 276(5315):1058-1065

[10] Saxena P., Hildemann L.M., Water-soluble organics in atmospheric particles: A critical review of the literature and application of thermodynamics to identify candidate compounds[J], J. Atmos. Chem., 1996,24(1):57-109

[11] Limbeck A., Puxbaum HOr., ganic acids in continental background aerosols[J], Atmos. Environ., 1999, 33: 1847-1852 
[12] Shi Y.Y., The electrochemical studies of atmospheric corrosion of typical metals[D]., Zhejiang University, 2008

[13] Liu Y.D. Influence of Behavior of Metallic Heat Transfer Surface on the Formation Characteristics of Crystalline Fouling[D]., Shandong University, 2008

[14] Bansal B., Chen X.D., Müller-Steinhagen H., Analysis of 'classical' deposition rate law for crystallization fouling[J], Chem. Eng. Process., 2008, 47:1201-1210

[15] Zhou X., Cai X.J., Liu X.C., et al., Analysis of heat exchanger fouling formation mechanism and influencing factors[J], Petro Chem. Equip., 2014, 43(1):84-88 